\title{
Field effect on surface states in a doped Mott-insulator thin film
}

\author{
D. Nasr Esfahani, ${ }^{*}$ L. Covaci, ${ }^{\dagger}$ and F. M. Peeters ${ }^{\ddagger}$ \\ Departement Fysica, Universiteit Antwerpen, Groenenborgerlaan 171, B-2020 Antwerpen, Belgium
}

(Received 24 June 2012; published 24 January 2013)

\begin{abstract}
Surface effects of a doped thin film made of a strongly correlated material are investigated both in the absence and presence of a perpendicular electric field. We use an inhomogeneous Gutzwiller approximation for a single-band Hubbard model in order to describe correlation effects. For low doping, the bulk value of the quasiparticle weight is recovered exponentially deep into the slab, but with increasing doping, additional Friedel oscillations appear near the surface. We show that the inverse correlation length has a power-law dependence on the doping level. In the presence of an electrical field, considerable changes in the quasiparticle weight can be realized throughout the system. We observe a large difference (as large as five orders of magnitude) in the quasiparticle weight near the opposite sides of the slab. This effect can be significant in switching devices that use the surface states for transport.
\end{abstract}

DOI: 10.1103/PhysRevB.87.035131

PACS number(s): 71.30.+h, 71.27.+a, 73.61.-r

\section{INTRODUCTION}

The metal insulator transition (MIT) based on carrier doping of a Mott insulator has been investigated experimentally and theoretically. ${ }^{1,2}$ Recently, the formation of a superconducting phase was observed at the interface of a Mott and band insulator, and the possible tuning of these transitions by an external electric field was reported. ${ }^{3}$ Moreover, a three-terminal setup was implemented by Son et al. who induced hole doping in a thin Mott insulator film from a doped band insulator through the application of a voltage difference between the drain and the gate terminals. ${ }^{4}$

For the above class of phenomena inhomogeneities and proximity effects play an essential role. In order to deal with such systems one needs a theoretical model that is able to include correlation effects in heterostructures while not being too computationally expensive such that one has the possibility to consider large enough system sizes. This is crucial, especially for the investigation of systems where surfaces and finite-size effects are significant, such as thin films made of strongly correlated materials. The interface between a band insulator and a strongly correlated system has been studied theoretically with a two-site dynamical mean field theory (DMFT) ${ }^{5}$ and the slave boson mean field theory (SBMFT). ${ }^{6}$ Such studies predict the formation of a two-dimensional electron gas at the interface which arises due to charge reconstruction. Surface correlation effects were studied theoretically in half-filled heterostructures modeled by a single-band Hubbard model. ${ }^{7}$ Also the penetration of metallic behavior into a Mott insulator was studied both within the Gutzwiller approximation and DMFT for the half-filled case. ${ }^{8,9}$ Surface correlation effects of a doped semi-infinite Hubbard model were investigated within an embedded DMFT for both single-band and multiband systems. ${ }^{10,11}$ Within this method, due to numerical limitations, only few surface layers (up to 6) can be used in order to address site dependent correlation effects. When the correlation length is large, this method is not reliable any more.

In order to describe position-dependent electronic correlation effects in a slab geometry we employ the Gutzwiller approximation (GA). While the GA works only for the metallic phase, it gives reliable information about the quasiparticle
(QP) weight of electrons at different spatial locations. For heterostructures, the GA was found to be in good qualitative agreement with the more refined DMFT method for the half-filled case. ${ }^{9}$ While the GA and SBMFT are equivalent for zero temperature, ${ }^{12}$ in two-site DMFT, like the GA, the bulk QP weight is governed by a simple power law and there is only a correction to $U_{c}$ when compared with the linearized DMFT. ${ }^{13,14}$ Generally, the GA overestimates the QP weight at all dopings but it is considered to be accurate enough to describe low-energy excitations and is routinely used for interpolations in combination with DMFT methods. ${ }^{2}$

The aim of this paper is to investigate the spatial dependence of the charge density and the QP weight of a doped correlated slab and to understand the correlation effects in the presence of an external electric field. We predict significant changes in the QP weight throughout the system. This study is motivated by potential applications in nanoscale switching devices with spatial controllable conductivity through the application of an external electric field.

The outline of the paper is as follows: after a brief derivation of the saddle-point equations for a slab geometry (Sec. II) the results for a doped correlated slab are presented in Sec. III A. Next the effect of an electric field is discussed in Sec. III B, and finally we present our conclusions in Sec. IV.

\section{MODEL AND METHOD}

The simplest Hamiltonian that is able to capture the essential physics of strongly correlated systems is the singleband Hubbard model, ${ }^{15}$

$$
\hat{H}_{U}=-\sum_{\langle i j\rangle \sigma} t_{i j} c_{i \sigma}^{\dagger} c_{j \sigma}+\sum_{i} U \hat{n}_{i \sigma} \hat{n}_{i \bar{\sigma}},
$$

where $t_{i j}$ are the hopping amplitudes, $\langle i j\rangle$ is summation over nearest-neighbor sites, and $U$ is the Hubbard energy describing the Coulomb interaction between two electrons with opposite spin located on the same site. In the presence of an external electric field the model becomes ${ }^{16}$

$$
\hat{H}=\hat{H}_{U}+\sum_{i \sigma} v_{i} \hat{n}_{i \sigma},
$$


where $v_{i}$ is the position-dependent potential. In spite of the simple form of the Hubbard model, exact solutions exist only for $d=1$ and $d=\infty, 1,17,18$ and therefore we are forced to work with approximations. If one is only concerned about ground-state properties or low-energy excitations, ${ }^{19}$ one of the choices is the Gutzwiller approximation (GA) which is the infinite dimension limit of the Gutzwiller wave function (GWF). ${ }^{18,20,21}$ The GWF is a many-body wave function with an additional degree of freedom used to reduce the weight of higher energy configurations. In the single-band Hubbard model these configurations are on-site double occupancies obtained when two electrons with opposite spin reside on the same site. The GWF is written as

$$
|\mathrm{GWF}\rangle=\prod_{i} \hat{P}_{i}\left|\phi_{0}\right\rangle
$$

where $i$ is the lattice site index, and the projector operators are defined as $\hat{P}_{i}=g_{e, i} \hat{e}_{i}+g_{\sigma, i} \hat{s}_{\sigma, i}+g_{\bar{\sigma}, i} \hat{s}_{\bar{\sigma}, i}+g_{d, i} \hat{d}_{i}$. The operators $\hat{e}=\left(1-\hat{n}_{i \sigma}\right)\left(1-\hat{n}_{i \bar{\sigma}}\right), \hat{s}_{\sigma}=\hat{n}_{i \sigma}\left(1-\hat{n}_{i \bar{\sigma}}\right)$, and $\hat{d}=$ $\hat{n}_{i \sigma} \hat{n}_{i \bar{\sigma}}$ are local projectors of zero, singly, and doubly occupied states, $\left|\varphi_{0}\right\rangle$ is a noninteracting Fermi sea and consists of both spin up and spin down states, and the $g$ coefficients are variational parameters. The following local constraints have to be satisfied in order to remove the local contributions in the diagramatic expansion of various expectation values: ${ }^{19,21}$

$$
\begin{aligned}
\left\langle\hat{P}_{i}^{\dagger} \hat{P}_{i}\right\rangle_{0} & =1, \\
\left\langle\hat{P}_{i}^{\dagger} \hat{P}_{i} c_{i \sigma}^{\dagger} c_{i \sigma}\right\rangle_{0} & =\left\langle c_{i \sigma}^{\dagger} c_{i \sigma}\right\rangle_{0},
\end{aligned}
$$

where $\langle\cdots\rangle_{0}$ represents the expectation value with respect to $\left|\varphi_{0}\right\rangle$. The explicit form of the above constraints is the following:

$$
\begin{gathered}
g_{i \sigma}{ }^{2}\left\langle\hat{e}_{i}\right\rangle_{0}+\sum_{\sigma} g_{i \sigma}{ }^{2}\left\langle s_{i \sigma}\right\rangle_{0}+g_{d, i}{ }^{2}\left\langle\hat{d}_{i}\right\rangle_{0}=1, \\
g_{i \sigma}{ }^{2}\left\langle s_{i \sigma}\right\rangle_{0}+g_{d, i}{ }^{2}\left\langle\hat{d}_{i}\right\rangle_{0}=\left\langle\hat{n}_{i \sigma}\right\rangle_{0} .
\end{gathered}
$$

In the limit of infinite dimensions the effect of the projectors $P_{i}$ requires the renormalization of the hopping amplitudes between different sites. ${ }^{18,21}$ These renormalization factors can be written as

$$
\sqrt{q_{i \sigma}}=\frac{g_{e, i} g_{\sigma, i} \sqrt{\left\langle\hat{e}_{i}\right\rangle_{0}\left\langle\hat{s}_{\sigma, i}\right\rangle_{0}}+g_{d, i} g_{\bar{\sigma}, i} \sqrt{\left\langle\hat{d}_{i}\right\rangle_{0}\left\langle\hat{s}_{\bar{\sigma}, i}\right\rangle_{0}}}{\left\langle n_{i \sigma}\right\rangle_{0}\left(1-\left\langle n_{i \sigma}\right\rangle_{0}\right)} .
$$

By substituting Eqs. (6) and (7) into Eq. (8) one arrives at an expression for $\sqrt{q}_{i \sigma}$ that is only a function of $g_{d, i},\left\langle\hat{n}_{i \sigma}\right\rangle_{0}$ and $\left\langle\hat{n}_{i \bar{\sigma}}\right\rangle_{0}$ as

$$
\sqrt{q_{i \sigma}}=\frac{\sqrt{\left(1-\left\langle\hat{n}_{i}\right\rangle_{0}+d_{i}\right)\left(\left\langle\hat{n}_{i \sigma}\right\rangle_{0}-d_{i}\right)}+\sqrt{d_{i}\left(\left\langle\hat{n}_{i \bar{\sigma}}\right\rangle_{0}-d_{i}\right)}}{\left\langle n_{i \sigma}\right\rangle_{0}\left(1-\left\langle n_{i \sigma}\right\rangle_{0}\right)},
$$

where $d_{i}=g_{d, i}^{2}\left\langle\hat{n}_{i \sigma}\right\rangle_{0}\left\langle\hat{n}_{i \bar{\sigma}}\right\rangle_{0}$ is the probability of double occupancy that is calculated within $|\mathrm{GWF}\rangle$ and $\left\langle\hat{n}_{i}\right\rangle_{0}=$ $\left\langle\hat{n}_{i \sigma}\right\rangle_{0}+\left\langle\hat{n}_{i \bar{\sigma}}\right\rangle_{0}$. Moreover, in addition to Eq. (8) the relation $\left\langle\hat{n}_{i \sigma}\right\rangle_{\text {Gutzwiller }}=\left\langle\hat{n}_{i \sigma}\right\rangle_{0}$ holds in the limit of infinite dimensions.
By considering the above relations, the total energy functional of an inhomogeneous system has the following form:

$$
\begin{aligned}
\langle\hat{H}\rangle_{\mathrm{GWF}}= & \sum_{\langle i j\rangle, \sigma}-t_{i j} \sqrt{q_{i \sigma}} \sqrt{q_{j \sigma}}\left\langle\hat{c}_{i \sigma}^{\dagger} \hat{c}_{j \sigma}\right\rangle_{0}+\sum_{i, \sigma} v_{i}\left\langle\hat{n}_{i \sigma}\right\rangle_{0} \\
& +\sum_{i} U g_{d, i}^{2}\left\langle\hat{n}_{i \sigma}\right\rangle_{0}\left\langle\hat{n}_{i \bar{\sigma}}\right\rangle_{0}
\end{aligned}
$$

The conditions $\langle\mathrm{GWF} \mid \mathrm{GWF}\rangle=\left\langle\varphi_{0} \mid \varphi_{0}\right\rangle$ and $\left\langle\varphi_{0} \mid \varphi_{0}\right\rangle=1$ are used in the above relation; the first relation itself is a consequence of the infinite dimensional limit and the second relation is just the normalization condition for $\left|\phi_{0}\right\rangle$.

Away from half-filling the problem of minimizing the energy functional is combersome because the renormalization factors, $q_{i \sigma}$, are functions of $\left\langle\hat{n}_{i \sigma}\right\rangle_{0}$. Therefore it is impossible to simply vary the above energy functional with respect to $\left\langle\phi_{0}\right|$. A possible approach, similar to DFT, is to start with an arbitrary value for $\left\langle\hat{n}_{i \sigma}\right\rangle_{0}$ and then to expand the energy functional as function of $\left\langle\hat{n}_{i \sigma}\right\rangle_{0}$ up to linear order around the starting $\left\langle\hat{n}_{i \sigma}\right\rangle_{0}$. This allows us to vary the energy functional with respect to $\left\langle\phi_{0}\right|$; moreover, this variation together with the normalization condition for $\left|\phi_{0}\right\rangle$ leads one to solve an eigenvalue problem, and a new value of $\left\langle\hat{n}_{i \bar{\sigma}}\right\rangle_{0}$ can be calculated by using the resulting wave function. This should be done until the desired convergence of the wave function or energy functional is achieved.

However, to alleviate this complication, instead of calculating the expectation value $\left\langle\hat{n}_{i \sigma}\right\rangle_{0}$, we introduce a set of new variational parameters $n_{i \sigma} \mathrm{s}$ that will play the role of local noninteracting occupancies (local noninteracting density matrices) which appear in the renormalization factors and double occupancies. It is then possible to let $n_{i \sigma}$ vary independently from $\left|\phi_{0}\right\rangle$. The energy functional that should be optimized has now the following form for a simple cubic slab geometry with periodic boundary conditions in the $x-y$ plane with free (001) surfaces:

$$
\begin{aligned}
\langle\hat{H}\rangle= & \sum_{i, k_{\|}, \sigma}\left(q_{i \sigma} \epsilon_{k_{\|}}+v_{i}\right)\left\langle\phi_{0}\left|\hat{c}_{i k_{\|} \sigma}^{\dagger} \hat{c}_{i k_{\|} \sigma}\right| \phi_{0}\right\rangle \\
& -\sum_{\langle i j\rangle k_{\|} \sigma} \sqrt{q_{i \sigma}} \sqrt{q_{j \sigma}} t\left\langle\phi_{0}\left|\hat{c}_{i k_{\|} \sigma}^{\dagger} \hat{c}_{j k_{\|} \sigma}\right| \varphi_{0}\right\rangle \\
& +\sum_{i \sigma} \lambda_{i \sigma}\left(\sum_{k_{\|}}\left\langle\phi_{0}\left|\hat{c}_{i k_{\|} \sigma}^{\dagger} \hat{c}_{i k_{\|} \sigma}\right| \phi_{0}\right\rangle-N_{k_{\|}} n_{i \sigma}\right) \\
& +\lambda\left(N_{k_{\|}} \sum_{i \sigma} n_{i \sigma}-N\right)+E\left(1-\left\langle\varphi_{0} \mid \varphi_{0}\right\rangle\right) \\
& +\sum_{i} N_{k_{\|}} U g_{d, i}^{2} n_{i \sigma} n_{i \bar{\sigma}},
\end{aligned}
$$

where $\epsilon_{k_{\|}}=-2 t\left(\cos k_{x}+\cos k_{y}\right)$; the Lagrange multipliers $\lambda_{i \sigma}$ are introduced to fix $n_{i \sigma}$ to $\left\langle\hat{n}_{i \sigma}\right\rangle_{0} . \Lambda$ is introduced to fix the total number of electrons, $E$ is considered to make sure that $\left|\varphi_{0}\right\rangle$ is normalized, $i$ and $j$ are index of layers in the $z$ direction, and $N_{k_{\|}}=N_{k_{x}} N_{k_{y}}$ is the total number of $k$ points. The optimization of the Lagrange function is performed through an iterative procedure, starting with a minimization with respect to $\left|\varphi_{0}\right\rangle$, which leads to a Schrödinger-like eigenvalue problem 
that has to be solved for each $k$ point:

$$
\begin{aligned}
& \sum_{i \sigma}\left(q_{i \sigma} \epsilon_{k_{\|}}+v_{i}+\lambda_{i \sigma}\right) \hat{c}_{i k_{\|} \sigma}^{\dagger} \hat{c}_{i k_{\|} \sigma}\left|\varphi_{0}\right\rangle \\
& \quad-\sum_{\langle i j\rangle \sigma} \sqrt{q_{i \sigma}} \sqrt{q_{j \sigma}} t \hat{c}_{i k_{\|} \sigma}^{\dagger} \hat{c}_{j k_{\|} \sigma}\left|\varphi_{0}\right\rangle=E_{k_{\|}}\left|\varphi_{0}\right\rangle,
\end{aligned}
$$

The resulting noninteracting many-body ground state energy and wave function are computed in the following way: $E_{N I}=\sum_{E_{k_{\|}, n}<E_{F}} E_{k_{\|}, n}$ and $\left|\varphi_{0}\right\rangle=\prod_{E_{k_{\|}, n}<E_{F}} \hat{c}_{k_{\|}, n \sigma}^{\dagger} \hat{c}_{k_{\|}, n \bar{\sigma}}^{\dagger}|0\rangle$, where $E_{F}$ is the Fermi energy and $n$ is the quantum number for the energy level of each $k$ point. The above noninteracting state $\left|\varphi_{0}\right\rangle$, which is now implicitly a function of all the variational parameters $\lambda_{i \sigma}, n_{i \sigma}, g_{i}$, and $\lambda$, should be inserted into Eq. (10), which becomes

$$
\begin{aligned}
\langle\hat{H}\rangle= & E_{N I}\left(n_{i \sigma}, \lambda_{i \sigma}, g_{d, i},\left|\varphi_{0}\right\rangle\right)-N_{k_{\|}} \sum_{i, \sigma} \lambda_{i \sigma} n_{i \sigma} \\
& +\lambda\left(N_{k_{\|}} \sum_{i, \sigma} n_{i \sigma}-N\right)+N_{k_{\|}} \sum_{i} U g_{d, i}^{2} n_{i \sigma} n_{i \bar{\sigma}} .
\end{aligned}
$$

In the next step we search for the stationary points of the above Lagrange function of a slab geometry for a paramagnetic system with $n_{i \sigma}=n_{i \bar{\sigma}}=n_{i}$ and $\left\langle\hat{n}_{i \sigma}\right\rangle_{0}=\left\langle\hat{n}_{i \bar{\sigma}}\right\rangle_{0}$ as

$$
\frac{\partial\langle\hat{H}\rangle}{\partial g_{d, i}}=2 \frac{\partial q_{i \sigma}}{\partial g_{d, i}}\left(\tilde{t}_{i}+\delta_{i, j \pm 1} \sqrt{\frac{q_{j \sigma}}{q_{i \sigma}}} \tilde{t}_{i j}\right)+2 N_{k_{\|}} U n_{i}{ }^{2} g_{i, d}=0,
$$

$$
\begin{aligned}
& \frac{\partial\langle\hat{H}\rangle}{\partial n_{i}}= 2 \frac{\partial q_{i \sigma}}{\partial n_{i}}\left(\tilde{t}_{i}+\delta_{i, j \pm 1} \sqrt{\frac{q_{j \sigma}}{q_{i \sigma}}} \tilde{t}_{i j}\right) \\
&+2 N_{k_{\|}}\left(\lambda-\lambda_{i}\right)+2 N_{k_{\|}} U g_{i, d}^{2} n_{i}=0, \\
& \frac{\partial\langle\hat{H}\rangle}{\partial \lambda_{i}}=2\left\langle\varphi_{0}\left|\sum_{k_{\|}} \hat{c}_{i k_{\|}}^{\dagger} \hat{c}_{i k_{\|} \sigma}\right| \varphi_{0}\right\rangle-2 N_{k_{\|}} n_{i}=0, \\
& \frac{\partial\langle\hat{H}\rangle}{\partial \lambda}=\left(N_{\text {total }}-2 N_{k_{\|}} \sum_{i} n_{i}\right)=0,
\end{aligned}
$$

where the spin index of renormalization factors and $\lambda_{i \sigma}$ is droped due to paramagnetic condition, $\tilde{t}_{i}=$ $\sum_{k_{\|}} \epsilon_{k_{\|}}\left\langle\varphi_{0}\left|\hat{c}_{i k_{\|} \sigma}^{\dagger} \hat{c}_{i k_{\|} \sigma}\right| \varphi_{0}\right\rangle, \tilde{t}_{i j}=-t \sum_{k_{\|}}\left\langle\varphi_{0}\left|\hat{c}_{i k_{\|} \sigma}^{\dagger} \hat{c}_{j k_{\|} \sigma}\right| \varphi_{0}\right\rangle$, and $N_{\text {total }}$ is the total number of electrons. $\tilde{t}_{i, i+1}$ and $\tilde{t}_{0,1}$ are equal to zero at the edge of the slab. For a detailed derivation of the saddle-point equations for an slab geometry in the paramagnetic case, we refer to Ref. [22]. This set of nonlinear equations can be solved by using a nonlinear solver based on Newton and/or quasi-Newton methods. Notice that $\left|\phi_{0}\right\rangle$ is still implicitly a function of the variational parameters and has to be updated again through Eq. (11) during the evaluations of the saddle-point equations throughout the optimization procedure. This means that we are all the time working with a $\left|\phi_{0}\right\rangle$ which satisfies the condition $\frac{\delta\langle\hat{H}\rangle}{\delta\left\langle\phi_{0}\right|}=0$.

It should be noticed that, together with the saddle point equations, the electrostatic forces due to long-range electronelectron and electron-ion interactions should be in principle considered. However, since the background permittivity of strongly correlated materials is usually very high, ${ }^{23}$ we tested the solutions with various high values of background permittivity and observed that long-range screening is negligible. ${ }^{22}$
We therefore set the value of the background permittivity to infinity in our calculations and ignore these effects. In order to numerically solve the set of saddle-point equations, we use a $16 \times 16$ Monkhorst-Pack ${ }^{24} k$ grid for which the total energy is well converged. We report results for $q_{i}$ as being the positiondependent QP weight, which is a measure of the mobility of the electrons within Fermi liquid theory. The inverse of the QP weight is proportional to the mass renormalization, which becomes divergent for $q_{i}=0$ corresponding to an insulating phase. ${ }^{25}$ The parameters $U$ and $v$ are scaled by the tight-binding parameter $t$. Throughout this work the thickness of the slab is taken as $L_{z}=90$ in units of the lattice constant.

\section{RESULTS}

\section{A. Hole doped correlated slab}

In Fig. 1(a) we depict the charge distribution near the surface for different values of doping and $U=16.2$, which is larger than the bulk critical $U$ for the half-filled case, i.e., $U_{C}^{\text {bulk,hf }}=16$. The surface region in which the charge density recovers its bulk value is doping dependent, resulting in the doping-dependent correlation length. Higher doping corresponds to lower correlation length. In the inset of Fig. 1(a) we present the charge transfer from the bulk to the surface $\left(n_{\text {surface }}-n_{\text {bulk }}\right)$. The doping dependence of this charge transfer is non-monotonic and is at maximum around

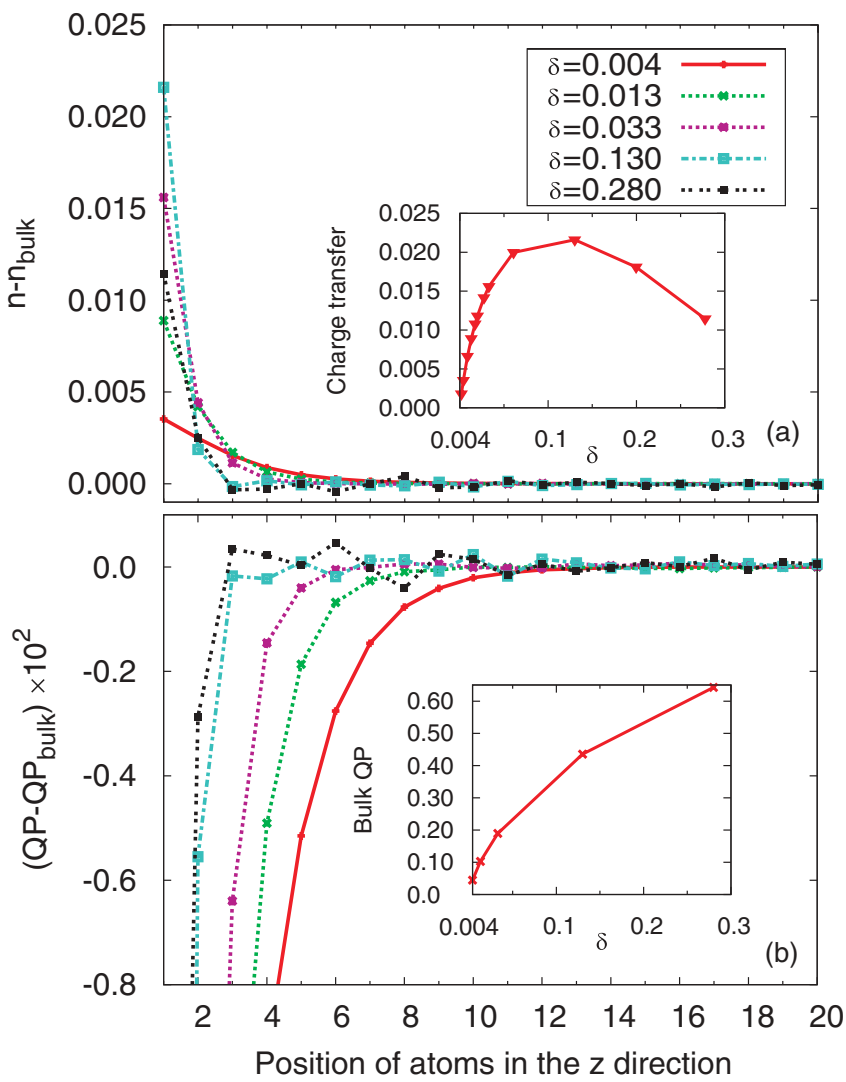

FIG. 1. (Color online) (a) Charge distribution for different dopings. Inset: Charge transfer from bulk to surface as a function of doping. (b) QP weight relative to the bulk QP weight near the surface for different dopings. The inset shows the doping dependence of the bulk QP weight. 
$\delta=0.15$. While our results for the charge transfer are in agreement with recent DMFT calculations for a hole-doped semi-infinite single-band Hubbard mode ${ }^{10}$ in the limit of large enough doping, our scaling analysis shows that considering only a few layers for the QP calculation may not be enough, specially for values of doping near half-filling for which the correlation length is larger that 6 lattice constants. In Fig. 1(b) the spatial-distribution QP weights $\left(q_{i}-q_{\text {bulk }}\right)$ are plotted for different values of doping and $U=16.2$. As in the half-filled case, ${ }^{7,9,22}$ the QP of electrons near the surface sites is suppressed due to the reduced coordination number together with the charge transfer to the surface sites from the bulk, which in turn results in a lack of kinetic energy and an enhancement of correlation effects. One can also observe Friedel oscillations which are more pronounced for higher doping due to lower correlation lengths. The inset of Fig. 1(b) shows the doping dependence of the bulk QP weight, $q_{\text {bulk }}$, which is in agreement with previous works and shows that, by increasing the doping, correlation effects are weaker. ${ }^{2}$ The correlation length can also be extracted from the spatial distribution of the QP weight near the surface. Similar to the dependence of the charge density, the QP weight recovers its bulk value within a characteristic length scale that depends on the correlation length. Friedel oscillations can also be observed but are suppressed for lower doping. Following Ref. 9 we observe that the spatial distribution of $\sqrt{q(x)}-\sqrt{q_{\text {bulk }}}$ is well fitted by an exponential decay for different values of the Hubbard repulsion and doping:

$$
\sqrt{q(x)}=\sqrt{q_{\text {bulk }}}+\left(\sqrt{q_{\text {surface }}}-\sqrt{q_{\text {bulk }}}\right) e^{-\frac{1}{\xi}(x-1)},
$$

where $\xi$ is the correlation length and $x$ the number of layer, starting from $x=1$. Since the correlation length $\xi$ depends on both $U$ and $\delta$, by fitting separately the spatial distribution of the QP weight we extract the corresponding correlation lengths. The results are summarized in Fig. 2, where $1 / \xi$ is plotted as a function of doping for different values of the Hubbard repulsion. We can extract a simple power-law dependence for the inverse correlation length: $\frac{1}{\xi}=A \delta^{\eta}$, with a mean-field-like

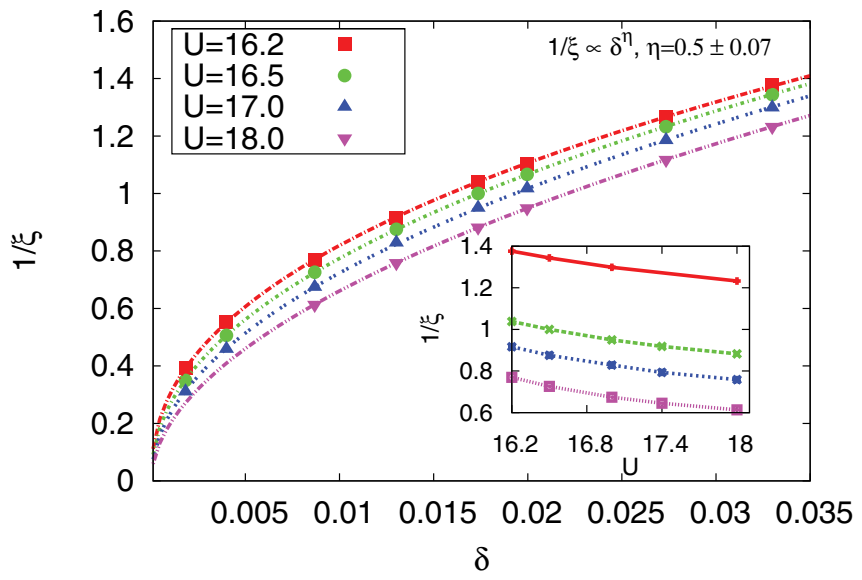

FIG. 2. (Color online) (a) Inverse correlation length as a function of doping for values of $U=16.2,16.5,17.0$, and 18.0. The inset shows the inverse of the correlation length as function of $U$ for four values of $\delta=0.009,0.013,0.017$, and 0.032 from bottom to top curves. exponent, ${ }^{8,26} \eta=0.5 \pm 0.07$, and a prefactor $A$ that is only a function of $U$. The inset of Fig. 2 shows the inverse correlation length as a function of $U$ for different dopings and, as expected, it is enhanced for higher Hubbard repulsions. The power-law dependence of the correlation length versus doping shows that for half-filling the correlation length diverges, which is a signature of the MIT that occurs for $U>U_{c}^{\text {bulk,hf }}$. A similar dependence of the correlation length versus Hubbard repulsion is observed for half-filling but when $U<U_{c}^{\text {bulk,hf }} .{ }^{26}$ In the latter case the criticality is governed by the Hubbard repulsion rather than the doping level.

\section{B. The effect of electric field}

The effect of an external electric field perpendicular to the slab on the spatial distribution of the QP weight is shown in Fig. 3 for $U=16.2, \delta=0.002$, and different values of the voltage difference. The inset shows the charge distribution for the same parameters. The main effect of the electric field is to redistribute the charges within the slab; however, in the strongly correlated regime when the Hubbard repulsion exceeds a certain crossover value, correlation effects enhance the charge transfer from less correlated sites to more correlated ones. This correlation-enhanced charge redistribution results in the accumulation of charges near the surface layers, bringing one side of the slab very close to half-filling. This effect is largest for $U>U_{c}^{\text {bulk,hf }}$.

To better clarify the correlation effects on the surface states of a correlated slab in the presence of an electric field, we depict in Fig. 4 the charge and quasiparticle (QP) distribution of a slab 90 layers thick and with a voltage difference $v=0.88$. The charge distribution for $U=15.22$ shows peaks near the surfaces, as expected; however, this behavior disappears for $U=15.74$ and $U=16.2$. This shows a clear crossover regime related to the enhancement of correlation effects. On the other hand the naive expectation, that the effect of an increased Hubbard repulsion is only to screen out the electric field, fails to explain the behavior of the system in the presence of the electric field in the strong-coupling regime. As shown in Fig. 4, by increasing the Hubbard repulsion,

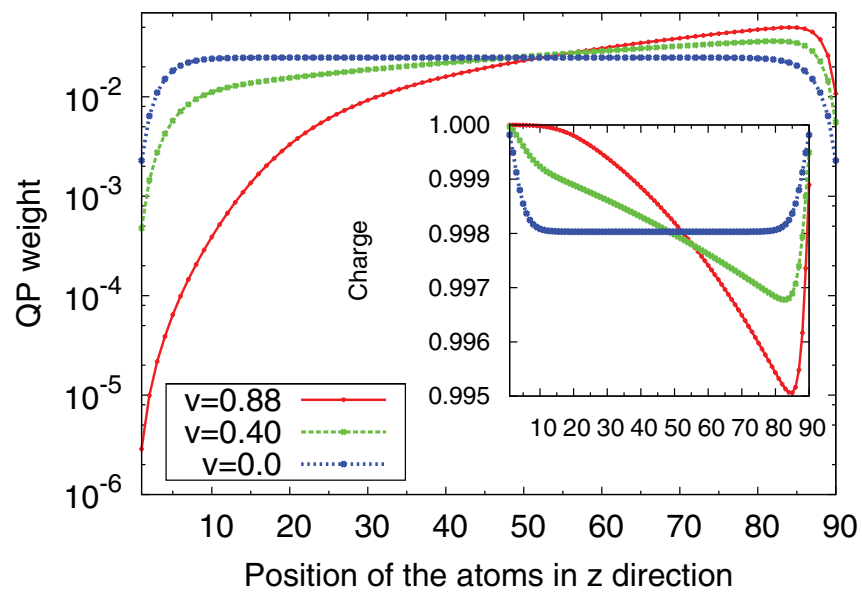

FIG. 3. (Color online) QP weight distribution and charge distribution (inset) for $U=16.2, \delta=0.002$, and three different values of the electric field. 




FIG. 4. (Color online) Charge distribution; the inset shows the charge transfer as a function of $U$ for fixed $v=0.88$ and $\delta=0.002$.

the charges do not go away from the surface but instead are accumulated at the surface. This mechanism of charge transfer from the places with enhanced delocalization to the places with enhanced correlations leads to a nontrivial enhancement of QP difference between the surfaces for large Hubbard repulsions. To further understand the charge redistribution enhancement due to correlation effects, we present in the inset of Fig. 4 the charge difference between the layers with highest charge density and the layers with lowest charge density as a function of $U$. This can be considered as a measure of the charge transfer throughout the system. As is clear from the inset of Fig. 4 there is a crossover value for $U$, given a fixed doping $\delta=0.002$ and voltage difference $v=0.88$. Above this value the effect of the $U$ plays a different role in the charge redistribution in the system. While below the crossover interaction the Hubbard repulsion competes with $v$ to prevent charge redistribution due to voltage difference, above the crossover it enhances the charge redistribution in favor of $v$. As is obvious from Fig. 3, the maximum QP weight is already achieved after a few layers from the surface on that side of the slab where the deviation of the charge density from half-filling is maximal. The reason that the QP weight is not maximal exactly at the surface is because of the suppression of the kinetic energy near the surface. On the other side of the slab, for larger electric fields the charge transfer assures that the charge density is near half-filling. Therefore, due to local correlation effects the QP weight is strongly suppressed. While the charge density near the surface is very close to half-filling (i.e., $n-1 \simeq 10^{-7}$ ) one may infer that the residual QP indicated in Fig. 3 for $x=1$ is mostly due to the proximity of the surface site to sites with higher QP weight rather than due to the local doping effect of these regions. In order to better understand the dependence of the QP weight on opposite sides of the slab, in Fig. 5 we show the QP weight for layers $x=1$ and $x=90$ as functions of voltage difference for three different values of doping.

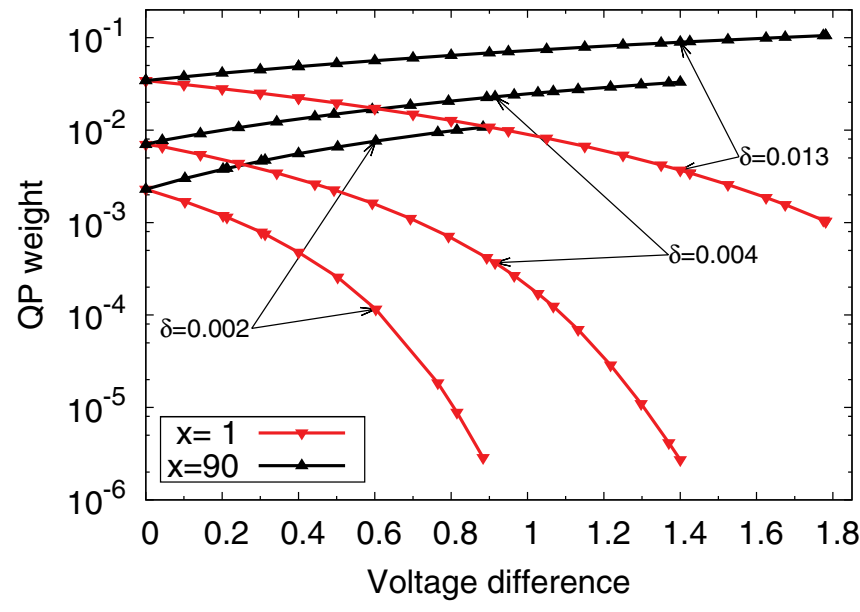

FIG. 5. (Color online) QP for $x=1$ and $x=90$ as functions of voltage difference for $U=16.2$ and three values of $\delta$.

The QP weights on the two surfaces differ by many orders of magnitude. For larger doping, higher electric fields are needed in order to achieve the same QP weight difference. This is because of the competing influence of doping and Hubbard repulsion on the correlation effects. The huge difference in QP weight near the two surfaces could be used for creating a transistor-like device made of strongly correlated materials. By using the surface states to conduct current one can simply switch on/off the device by switching the polarity of the gate. Thus, turning on/off the electric conduction is now a consequence of the surface resistance ratio of the two sides.

\section{CONCLUSIONS}

By using an inhomogeneous Gutzwiller approach applied to the paramagnetic single-band Hubbard model for a slab geometry, we described a hole-doped Mott thin film. In the absence of applied electric field we calculated the positiondependent charge density and QP weight and showed that the inverse correlation length has a power-law dependence on doping.

When a perpendicular electric field is applied, charges will accumulate on one side of the slab. This correlation enhanced charge redistribution will in turn induce a large difference in the QP weight on the two sides of the slab, which was found to be as large as five orders of magnitude. We propose that a three-terminal device with surface contacts can take advantage of this effect. For resistance switching purposes one would expect large on/off ratios of surface resistances when the electric field switches polarity.

\section{ACKNOWLEDGMENTS}

This work was supported by the Flemish Science Foundation (FWO-Vl).

\footnotetext{
*Davoud.NasrEsfahani@ua.ac.be

†lucian@covaci.org

${ }^{\ddagger}$ Francois.Peeters@ua.ac.be

${ }^{1}$ A. Georges, G. Kotliar, W. Krauth, and M. J. Rozenberg, Rev. Mod. Phys. 68, 13 (1996).
}

${ }^{2}$ G. Kotliar, S. Y. Savrasov, K. Haule, V. S. Oudovenko, O. Parcollet, and C. A. Marianetti, Rev. Mod. Phys. 78, 865 (2006).

${ }^{3}$ A. D. Caviglia, S. Gariglio, N. Reyren, and D. Jaccard, Nature (London) 456, 624 (2008). 
${ }^{4}$ J. Son, S. Rajan, S. Stemmer, and S. James Allen, J. Appl. Phys. 110, 084503 (2011).

${ }^{5}$ S. Okamoto and A. J. Millis, Phys. Rev. B 70, 241104(R) (2004).

${ }^{6}$ A. Ruegg, S. Pilgram, and M. Sigrist, Phys. Rev. B 75, 195117 (2007).

${ }^{7}$ M. Potthoff and W. Nolting, Phys. Rev. B 59, 2549 (1999).

${ }^{8}$ R. W. Helmes, T. A. Costi, and A. Rosch, Phys. Rev. Lett. 101, 066802 (2008).

${ }^{9}$ G. Borghi, M. Fabrizio, and E. Tosatti, Phys. Rev. B 81, 115134 (2010).

${ }^{10}$ R. Nourafkan and F. Marsiglio, Phys. Rev. B 83, 155116 (2011).

${ }^{11}$ R. Nourafkan and F. Marsiglio, Phys. Rev. B 84, 075133 (2011).

${ }^{12}$ J. Bunemann and F. Gebhard, Phys. Rev. B 76, 193104 (2007).

${ }^{13}$ M. Potthoff, Phys. Rev. B 64, 165114 (2001).

${ }^{14}$ X. Y. Zhang, M. J. Rozenberg, and G. Kotliar, Phys. Rev. Lett. 70, 1666 (1993).

${ }^{15}$ J. Hubbard, Proc. R. Soc. London 276, 238 (1963).
${ }^{16}$ M. Graf and P. Vogl, Phys. Rev. B 51, 4940 (1995).

${ }^{17}$ E. Lieb and F. Y. Wu, Phys. Rev. Lett. 20, 1445 (1968).

${ }^{18}$ W. Metzner and D. Vollhardt, Phys. Rev. Lett. 62, 324 (1989).

${ }^{19}$ J. Bunemann, F. Gebhard, and R. Thul, Phys. Rev. B 67, 075103 (2003).

${ }^{20}$ M. C. Gutzwiller, Phys. Rev. Lett. 10, 159 (1963).

${ }^{21}$ F. Gebhard, Phys. Rev. B 41, 9452 (1990).

${ }^{22}$ D. Nasr Esfahani, L. Covaci, and F. M. Peeters, Phys. Rev. B 85, 085110 (2012).

${ }^{23}$ P. Lunkenheimer, S. Krohns, S. Riegg, S. G. Ebbinghaus, A. Reller, and A. Loidl, Eur. Phys. J.: Special Topics 180, 61 (2009).

${ }^{24}$ H. J. Monkhorst and J. D. Pack, Phys. Rev. B 12, 15 (1976).

${ }^{25} \mathrm{P}$. Fazekas, Lecture Notes on Electron Correlations and Magnetism, Series in Modern Condensed Matter Physics, Vol. 5 (World Scientific, Singapore, 1999).

${ }^{26}$ G. Borghi, M. Fabrizio, and E. Tosatti, Phys. Rev. Lett. 102, 066806 (2009). 